\title{
Hepatitis B virus $X$ protein and hypoxia-inducible factor-1 $\alpha$ stimulate Notch gene expression in liver cancer cells
}

\author{
SHENG-LI YANG ${ }^{1}$, QUAN-GUANG REN ${ }^{1}$, TAO ZHANG $^{1}$, XIAOLI PAN ${ }^{2}$, LU WEN $^{1}$, \\ JIAN-LI HU ${ }^{1}$, CHAO YU ${ }^{3}$ and QIAN-JIN HE ${ }^{4}$ \\ ${ }^{1}$ Cancer Center and ${ }^{2}$ Department of Gastroenterology and Hepatology, Union Hospital, Tongji Medical College, \\ Huazhong University of Science and Technology, Wuhan, Hubei 430022; ${ }^{3}$ Department of Hepatobiliary Surgery, \\ Affiliated Hospital of Guiyang Medical College, Guiyang, Guizhou 550001; ${ }^{4}$ Department of General Surgery, \\ Huanggang Central Hospital, Huanggang, Hubei 438000, P.R. China
}

Received May 6, 2016; Accepted June 17, 2016

DOI: $10.3892 /$ or.2016.5211

\begin{abstract}
Increasing evidence has demonstrated that Notch genes, including Notch1, Notch2, Notch3 and Notch4, are involved in carcinogenesis. However, the expression and regulation of Notch genes in hepatocellular carcinoma (HCC) tissues have not been fully investigated. In the present study, immunohistochemical and quantitative real-time PCR (qPCR) analyses were performed to examine the expression of Notch genes in normal human liver, HBV-related HCC and paired peritumoral tissues. Additionally, qPCR and western blotting were utilized to investigate the impact of hepatitis $\mathrm{B}$ virus $\mathrm{X}$ protein $(\mathrm{HBx})$ and hypoxia-inducible factor- $1 \alpha(\mathrm{HIF}-1 \alpha)$ on the regulation of Notch gene expression. The immunohistochemical and qPCR results showed that the expression levels of Notch1, Notch3 and Notch4 were significantly higher in HCC tissues than the levels in peritumoral and normal liver tissues. However, no significant difference in Notch2 expression was found between HCC and peritumoral tissues. Among the four Notch genes, immunohistochemical analyses found that only the increased level of Notch 3 in HCC tissues was positively correlated with vascular invasion of liver cancer $(\mathrm{P}<0.05)$. Moreover, we found that overexpression of both $\mathrm{HBx}$ and HIF-1 $\alpha$ increased the expression of Notch1, Notch3 and Notch4 in HepG2 and Bel-7404 cell lines. In summary, the present study demonstrated that Notch1, Notch3 and Notch4 were upregulated in HCC tissues and that HBx and HIF-1 $\alpha$ may be the factors that cause the overexpression of Notch genes. Furthermore, the increased expression of Notch3 was closely related to the vascular invasiveness of HCC.
\end{abstract}

Correspondence to: Professor Jian-Li Hu, Cancer Center, Union Hospital, Tongji Medical College, Huazhong University of Science and Technology, 1277 Jiefang Avenue, Wuhan, Hubei 430022, P.R. China

E-mail: j15199@126.com

Key words: hepatitis B virus X protein, hypoxia-inducible factor-1 $\alpha$, Notch, hepatocellular carcinoma

\section{Introduction}

In recent years, acceptance and application of the theory concerning tumor stem cells are gaining impetus. Tumor stem cells are responsible for tumor occurrence, relapse, metastasis and drug resistance. Inhibition and blocking of the proliferation of tumor stem cells can suppress the growth of tumors (1). Proliferation and stemness maintenance of tumor stem cells are related to the Notch, Wnt and Hedgehog signaling pathways $(2,3)$. Among them, the Notch signaling pathway is the simplest and consists of only three parts, Notch receptor, Notch ligand and intracellular effector (DNA-binding protein CSL, $\mathrm{CBFl} /$ suppressor of hairless/Lag1). When the Notch signaling pathway is activated, the Notch receptor binds to the ligand and is then cleaved by the protein convertase $(2,3)$. The Notch intracellular signaling domain (NICD) is released into the cytoplasm and enters the nucleus where it binds to the transcriptional factor CSL, forming the NICD/CSL complex. As a result, the target genes HES, HEY and HERP are activated $(2,3)$.

The Notch receptor family includes four members, Notch1, Notch2, Notch3 and Notch4, which play different roles in tumor formation $(4,5)$. Divergent conclusions have been drawn concerning the relationships of the four Notch receptors to liver cancer, and no scientific consensus has yet been reached concerning the reason for the abnormal expression levels of Notch1, Notch2, Notch3 and Notch4 in liver cancer (5). Various studies have shown that the hepatitis B virus X protein $(\mathrm{HBx})$ activates the Notch1 signaling pathway (6-8), whereas Xu et al indicated that HBx inhibits this pathway (9). Hypoxia-inducible factor- $1 \alpha$ (HIF-1 $\alpha$ ) is known to regulate Notch1 expression (10-12), but its effect on other Notch receptors is uncertain, which we aimed to clarify in the present study.

\section{Materials and methods}

Patients and clinicopathologic information. HBV-positive HCC tissues $(\mathrm{n}=105)$ and their paired peritumoral tissues $(\mathrm{n}=52)$, as well as normal liver samples from hepatic hemangioma $(n=10)$ were sectioned for immunohistochemical analysis (IHC). Among these samples, 52 HCC and paired 
Table I. PCR primers and conditions.

\begin{tabular}{|c|c|c|c|}
\hline Gene & Primers & Temperature $\left({ }^{\circ} \mathrm{C}\right)$ & Product size (bp) \\
\hline Notch1 & $\begin{array}{l}\text { 5'-GTCAACGCCGTAGATGACC-3' } \\
\text { 5'-TTGTTAGCCCCGTTCTTCAG-3' }\end{array}$ & 61.4 & 101 \\
\hline Notch2 & $\begin{array}{l}\text { 5'-ACTGTGAGGAGCAACTCGAT-3' } \\
\text { 5'-TCCACTTCATACTCACAGTTGA-3' }\end{array}$ & 53.5 & 133 \\
\hline Notch3 & $\begin{array}{l}\text { 5'-TGACCGTACTGGCGAGACT-3' } \\
\text { 5'-CCGCTTGGCTGCATCAGCA-3' }\end{array}$ & 62.1 & 67 \\
\hline Notch4 & $\begin{array}{l}\text { 5'-AACTCСТCCCCAGGAATCTG-3' } \\
\text { 5'-ССТCCATCCAGCAGAGGTT-3' }\end{array}$ & 57.5 & 168 \\
\hline$\beta$-actin & $\begin{array}{l}\text { 5'-AGTTGCGTTACACCCTTTCTTGAC-3' } \\
\text { 5'-GCTCGCTCCAACCGACTGC-3' }\end{array}$ & 63.9 & 171 \\
\hline $\mathrm{HIF}-1 \alpha$ & $\begin{array}{l}\text { 5'-CATCTCCATCTCCTACCCACA-3' } \\
\text { 5'-CTTTTCCTGCTCTGTTTGGTG-3' }\end{array}$ & 58.3 & 105 \\
\hline
\end{tabular}

HIF- $1 \alpha$, hypoxia-inducible factor $1 \alpha$.

peritumoral tissues were collected from The Center Hospital of Huanggang (Huanggang, China) between June 2008 and June 2011. The rest of the samples were collected from the Affiliated Hospital of Guiyang Medical College (Guiyang, China) from November 2006 to July 2010. All the human studies were approved by the Human Ethics Committee of The Center Hospital of Huanggang and Guiyang Medical College. All tissue samples were collected from patients before any medical treatment. Peritumoral tissues were obtained at least $2 \mathrm{~cm}$ away from the primary site of the tumor. All HCC patients tested positive for $\mathrm{HBV}$ antigen (HBsAg) and negative for the antibodies against hepatitis $\mathrm{C}$ virus (anti-HCV) and human immunodeficiency virus (anti-HIV).

Reagents and cell lines. Goat anti-human Notch1 polyclonal (SC-6014), goat anti-human Notch2 polyclonal (SC-32346), rabbit anti-human Notch3 polyclonal (SC-5593), rabbit antihuman Notch4 polyclonal (SC-5594), mouse anti-human monoclonal antibodies against HIF-1 $\alpha$ (SC-53546) and mouse monoclonal antibodies raised against baculovirus expressed recombinant Hep B xAg (SC-57760) were purchased from Santa Cruz Biotechnology (Santa Cruz, CA, USA). The human HCC cell line HepG2 was purchased from the American Type Culture Collection (ATCC; Rockville, MD, USA). Another HCC cell line Bel-7404 was purchased from the Institute of Cell Biology, SIBS, CAS (Shanghai, China). HIF-1 $\alpha$ expression plasmids and the corresponding control plasmids (pcDNA3.1), as well as HBx expression and their control plasmids (pEGFP-N1) were purchased from Shanghai GeneChem (Shanghai, China).

IHC. IHC was performed as previously described (13). All the primary antibodies for the Notch genes were diluted at 1:50. The protein expression of Notch1, Notch2, Notch3 and Notch4 was quantitated by the number of cells exhibiting cytoplasmic and nuclear staining and scored according to the standard developed by Gao et al (14). Briefly, a percent intensity (PI) score was given based on the percentage of stained cells in each cellular compartment: score 0 for $0 \%$ cell staining, score 1 for $1-25 \%$, score 2 for $26-50 \%$, score 3 for $51-75 \%$, and score 4 for $76-100 \%$. Next, the staining intensity (I) was scored for the tissues: score 0 for no staining, score 1 for weak but definite staining, score 2 for moderate staining, score 3 for pronounced staining, and score 4 for intense staining. The immunoreaction score (IRS) was calculated by the formula IRS = PI x I/4. If one IRS score was greater than the median of all the scores, it was categorized as high protein expression. Otherwise, it was categorized as low protein expression.

Quantitative real-time PCR ( $P P C R)$ and western blotting. TRIzol reagents (Invitrogen, Carlsbad, CA, USA) were used to isolate total RNA from 150-200 mg of 52 fresh frozen HCC tumor and their adjacent liver tissues. RNA extraction, cDNA synthesis, qPCR reaction and western blotting were performed as previously reported (15). The primer sequences used in the present study are listed in Table I. All the primary antibodies for Notch1-4 were diluted at 1:250.

Promoter analysis. The TRANSFAC software (http://www. biobase.international.com) was used to identify potential HIF-1 $\alpha$ binding sites in the promoter regions of Noth1-4 following the software's instructions.

Statistical analysis. The statistical analyses were performed using SPSS software (version 20.0; SPSS, Inc., Chicago, IL, USA). Paired t-test, Mann-Whitney U test, or one-way ANOVA were used. A P-value $<0.05$ was considered statistically significant. Results are expressed as mean \pm SD of three independent experiments.

\section{Results}

Expression of Notch1, Notch2, Notch3 and Notch4 in HCC tissues. It has been reported that the four Notch members, 
A
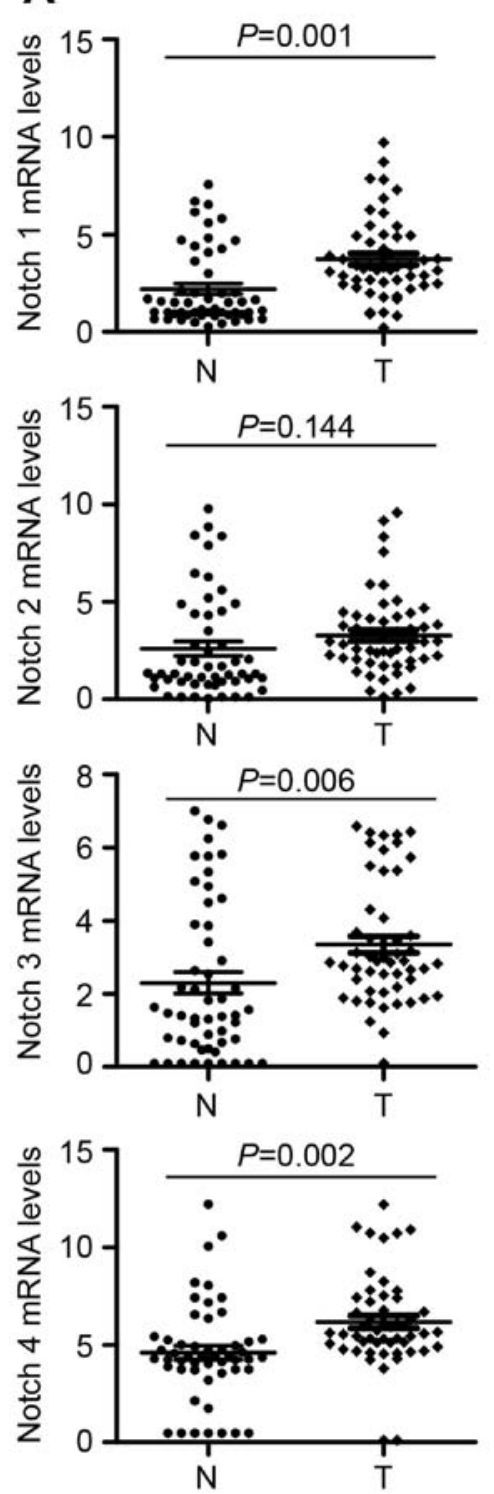

B
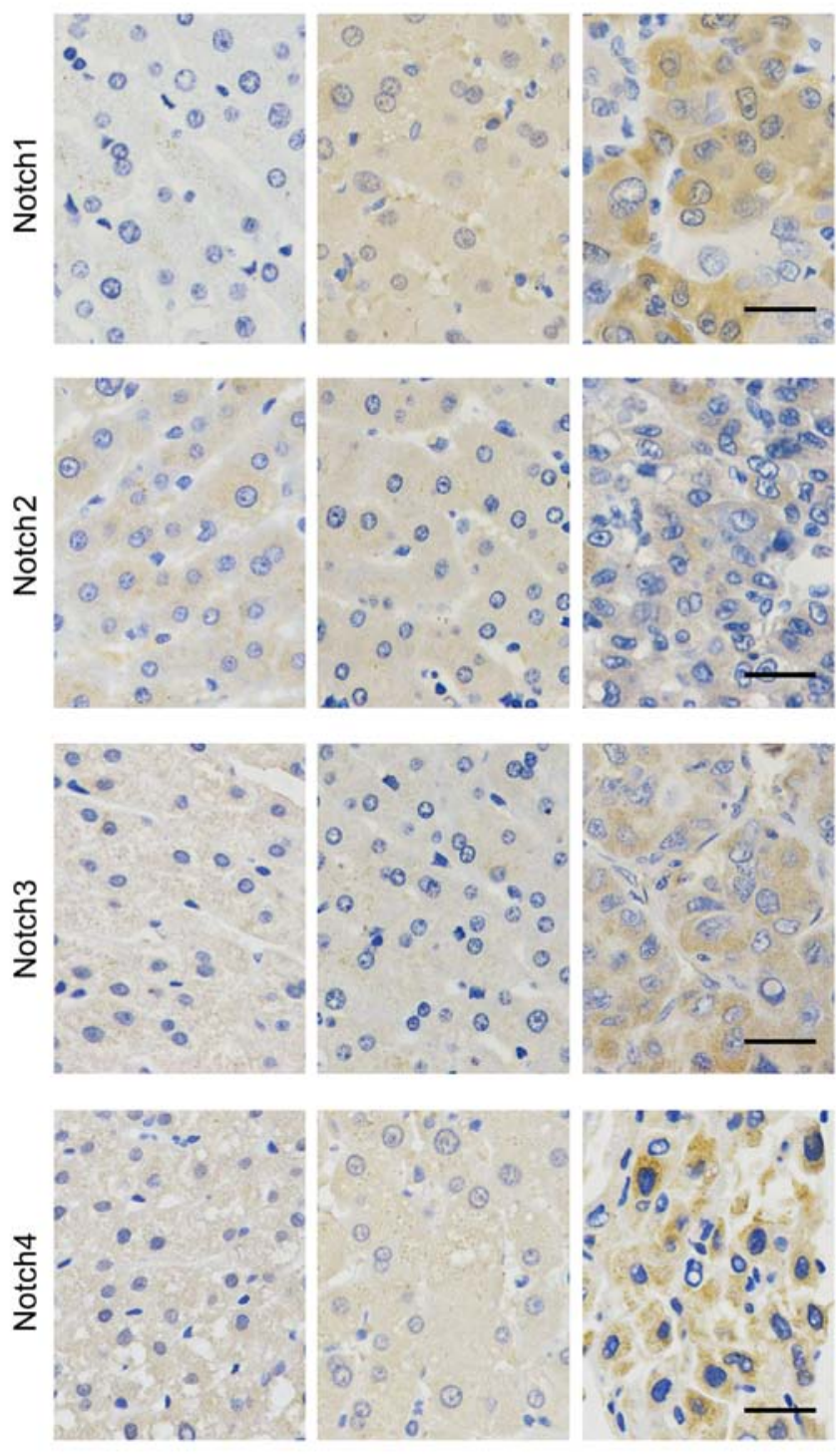

Figure 1. mRNA and protein expression of Notch genes in HCC tumors and their peritumoral tissues. (A) Quantitative real-time PCR (qPCR) results show increased mRNA expression of Notch1, 3 and 4 in HCC tumor tissues (T) compared to peritumoral tissues (N). (B) Immunohistochemical analysis also found enhanced staining intensity of Notch1, 3 and 4 proteins in HCC tumor tissues compared to their intensities in normal liver and peritumoral tissues. Scale bar, $10 \mu \mathrm{m}$.

Notch1-4, are closely associated with the development of many types of cancers $(16,17)$. To investigate the relationship of Notch gene expression and HCC development, we compared the expression of Notch1, Notch2, Notch3 and Notch4 in HBV-positive HCC tissues with their expression in paired peritumoral tissues. The qPCR results showed that the mRNA expression of Notch1, Notch3 and Notch4 was significantly higher in HCC tissues than that in peritumoral tissues (Fig. 1A). However, there was no statistically significant difference in Notch 2 mRNA expression between the HCC and peritumoral tissues (Fig. 1A). In addition to qPCR, IHC was performed to detect the protein expression of Notch1-4 in 105 HCC samples, 52 paired peritumoral specimens and 10 normal liver tissues. The results indicated that Notch1-4 were all expressed in the cells of HCC, peritumoral and normal liver tissues with varied intensity. As shown in Fig. 1B, the protein expression of Notch1, Notch3 and Notch4 in HCC tissues was markedly higher than that in the peritumoral and normal liver tissues. However, no difference in Notch2 protein expression was observed among the three types of tissues (Fig. 1B).

Clinical significance of altered Notch gene expression in HCC tissues. Next, we studied the relationship between Notch gene expression and the clinicopathological features of HCC patients. We found that the expression of Notch3 in the HCC tissues with vascular invasion was significantly higher than that in the HCC tissues without vascular invasion $(\mathrm{P}<0.05$; Table II). However, no significant correlation was found between Notch3 expression and the other clinicopathological features, including age, gender, presence of cirrhosis, tumor size, serum $\alpha$-fetoprotein (AFP) level and grade of tumor differentiation. In addition, no correlation was observed 
A

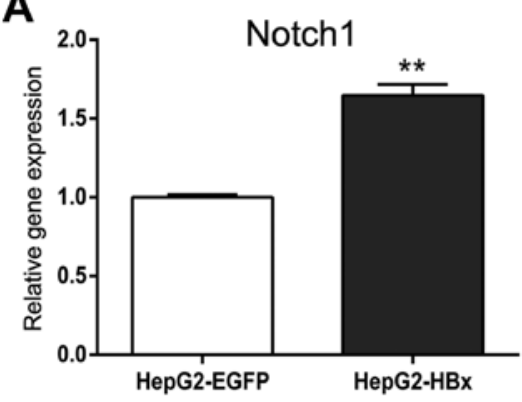

B

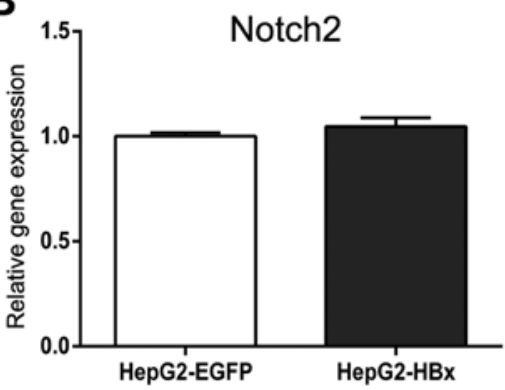

C

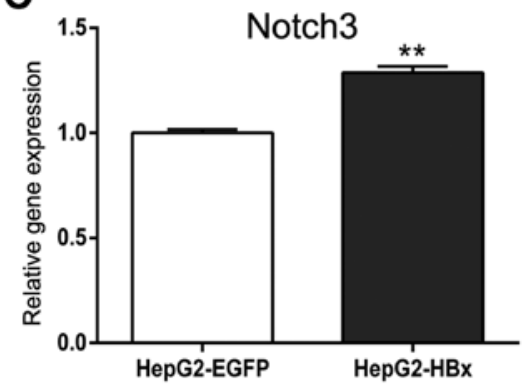

D

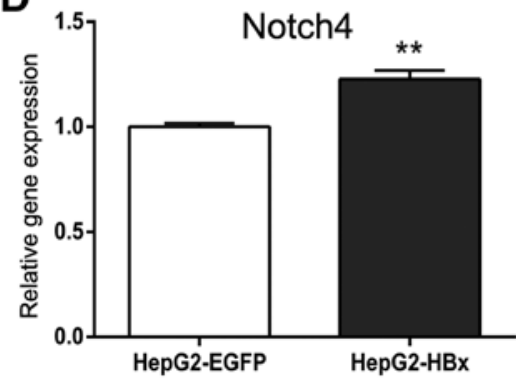

$\mathbf{E}$

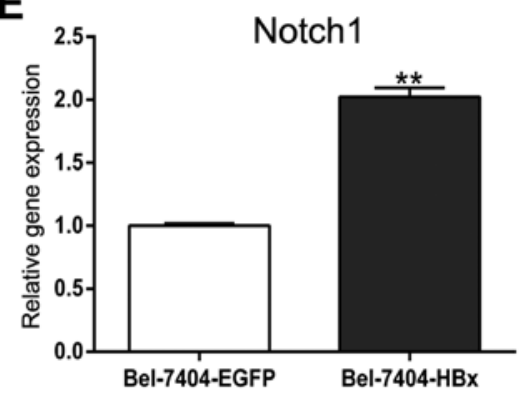

$\mathbf{F}$

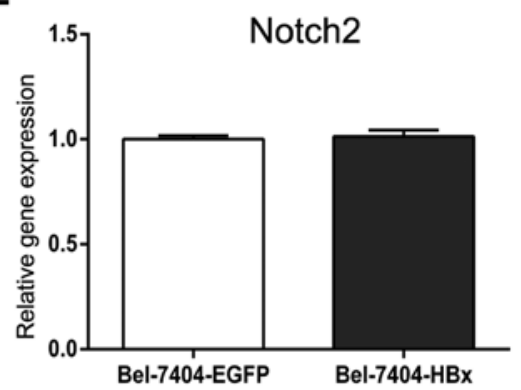

G

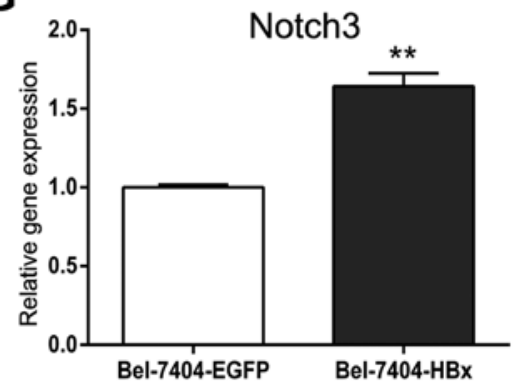

H

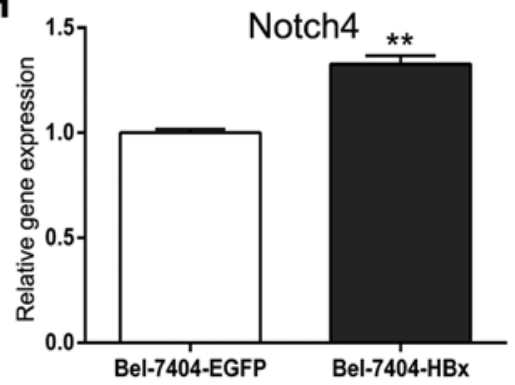

Figure 2. HBx increases the mRNA expression of Notch1, 3 and 4 in HCC cell lines. qPCR results show that the mRNA expression of Notch1, 3 and 4 was increased in the HepG2 cells (A, C and D) and Bel-7404 cells (E, G and H) transfected with the HBx expression plasmids compared with their corresponding control cells transfected with an empty vector (EGFP). The effect of transfecting HBx expression plasmids into (B) HepG2 and (F) Bel-7404 cells on the mRNA expression of Notch 2 resulted in no significant differences, compared to the control cells, respectively. Results are presented as mean $\pm \mathrm{SE}\left({ }^{* *} \mathrm{P}<0.01\right)$.

between the expression of Notch1, Notch2 and Notch4 and all the documented clinicopathological features (Table II). These findings suggest that Notch3 is a valuable predictor for early invasion and metastasis of HCC.

HBx is involved in the upregulation of Notch1, Notch3 and Notch4 in HCC. To investigate the molecular mechanism leading to the upregulation of Notch1, Notch3 and Notch4 in HCC tissues, we examined the role of HBx, a gene closely associated with HCC development, in controlling the expression of Notch1-4. We found that the HepG2 and Bel-7404 cells transfected with HBx expression plasmids had elevated mRNA and protein levels of Notch1, Notch3 and Notch4 compared to the levels in the cells transfected with the control plasmids (Figs. 2 and 3). However, there was no significant alteration in Notch 2 expression at either the mRNA or protein level following the overexpression of $\mathrm{HBx}$. $\mathrm{HBx}$ has been reported to be upregulated in HCC tissues (18), and therefore, it may be one of the factors that lead to the increased expression of Notch1, Notch3 and Notch4 in HCC tissues.

HIF-1 $\alpha$ is involved in the upregulation of Notch1-4 in HCC. It has been widely reported that HIF-1 $\alpha$ increases the expression of Notch1 in many types of human cancer cells (10-12). However, it remains unclear concerning whether HIF-1 $\alpha$ is involved in regulating the expression of Notch2, Notch3 and 
Table II. Correlations of Notch protein expression in surgical specimens of HCC with clinicopathological characteistics of the HCC cases.

\begin{tabular}{|c|c|c|c|c|c|c|c|c|c|c|c|c|}
\hline \multirow[b]{2}{*}{ Parameters } & \multicolumn{2}{|c|}{ Notch1 } & \multirow[b]{2}{*}{ P-value } & \multicolumn{2}{|c|}{ Notch2 } & \multirow[b]{2}{*}{ P-value } & \multicolumn{2}{|c|}{ Notch3 } & \multirow[b]{2}{*}{ P-value } & \multicolumn{2}{|c|}{ Notch4 } & \multirow[b]{2}{*}{ P-value } \\
\hline & Low & High & & Low & High & & Low & High & & Low & High & \\
\hline Age (years) & & & 0.537 & & & 0.181 & & & 0.625 & & & 0.103 \\
\hline$<50$ & 17 & 15 & & 13 & 19 & & 15 & 17 & & 12 & 20 & \\
\hline$\geq 50$ & 34 & 39 & & 40 & 33 & & 38 & 35 & & 40 & 33 & \\
\hline Gender & & & 0.190 & & & 0.861 & & & 0.527 & & & 0.777 \\
\hline Male & 36 & 44 & & 40 & 40 & & 39 & 41 & & 39 & 41 & \\
\hline Female & 15 & 10 & & 13 & 12 & & 14 & 11 & & 13 & 12 & \\
\hline Cirrhosis & & & 0.639 & & & 0.285 & & & 0.064 & & & 0.375 \\
\hline Absence & 25 & 24 & & 22 & 27 & & 20 & 29 & & 22 & 27 & \\
\hline Presence & 26 & 30 & & 31 & 25 & & 33 & 23 & & 30 & 26 & \\
\hline Tumor size $(\mathrm{cm})$ & & & 0.137 & & & 0.917 & & & 0.498 & & & 0.375 \\
\hline$<5$ & 20 & 29 & & 25 & 24 & & 23 & 26 & & 22 & 27 & \\
\hline$\geq 5$ & 31 & 25 & & 28 & 28 & & 30 & 26 & & 30 & 26 & \\
\hline $\operatorname{AFP}(\mu \mathrm{g} / \mathrm{l})$ & & & 0.473 & & & 0.285 & & & 0.776 & & & 0.285 \\
\hline$<400$ & 30 & 28 & & 32 & 26 & & 30 & 28 & & 26 & 32 & \\
\hline$\geq 400$ & 21 & 26 & & 21 & 26 & & 23 & 24 & & 26 & 21 & \\
\hline Histological grade & & & 0.624 & & & 0.248 & & & 0.670 & & & 0.272 \\
\hline Well & 14 & 11 & & 15 & 10 & & 14 & 11 & & 13 & 12 & \\
\hline Moderate & 24 & 30 & & 23 & 31 & & 25 & 29 & & 23 & 31 & \\
\hline Poor & 13 & 13 & & 15 & 11 & & 14 & 12 & & 16 & 10 & \\
\hline Vascular invasion & & & 0.948 & & & 0.230 & & & 0.034 & & & 0.275 \\
\hline Absence & 39 & 41 & & 43 & 37 & & 45 & 35 & & 42 & 38 & \\
\hline Presence & 12 & 13 & & 10 & 15 & & 8 & 17 & & 10 & 15 & \\
\hline
\end{tabular}

HCC, hepatocellular carcinoma; AFP, $\alpha$-fetoprotein.

A

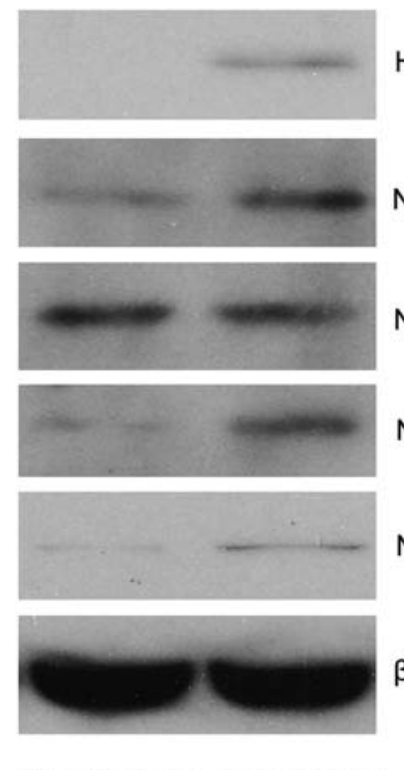

HepG2-EGFP HepG2-HBx
B

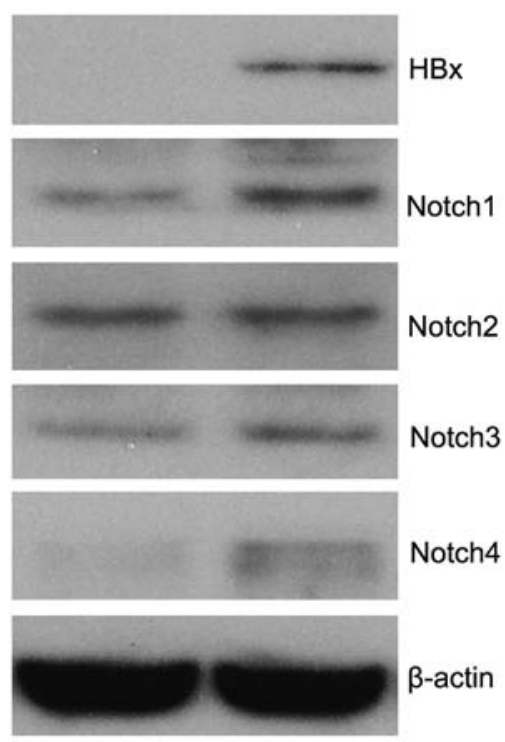

Bel-7404-EGFP Bel-7404-HBx

Figure 3. HBx increases the protein expression of Notch1, 3 and 4 in HCC cell lines. Western blotting was used to detect elevated protein expression of Notch1, 3 and 4 in the (A) HepG2 and (B) Bel-7404 cells transiently transfected with HBx expression plasmids compared to their corresponding control cells transfected with an empty vector (EGFP). $\beta$-actin was used as a loading control. 
A

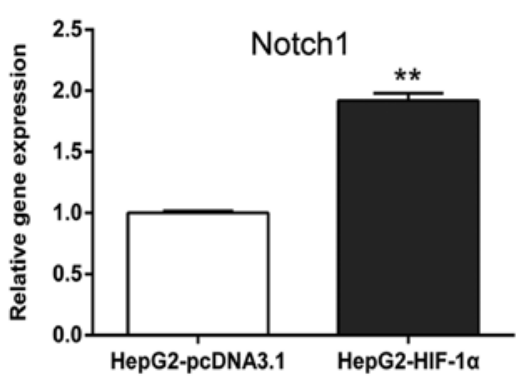

B

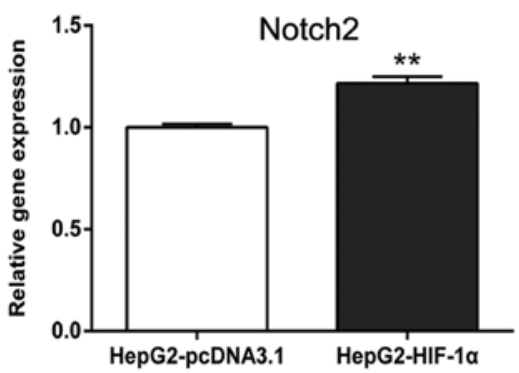

C

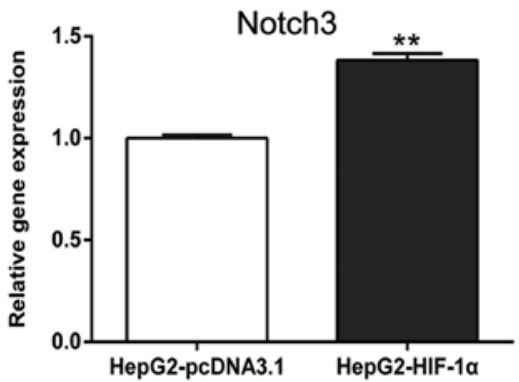

D

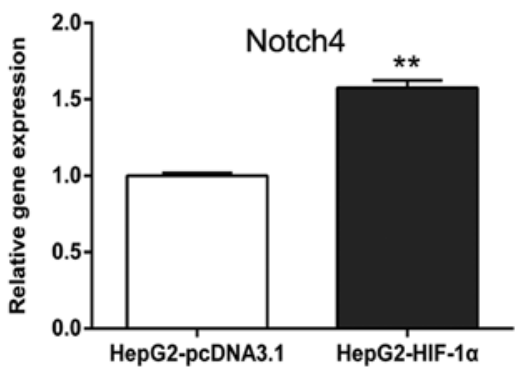

E

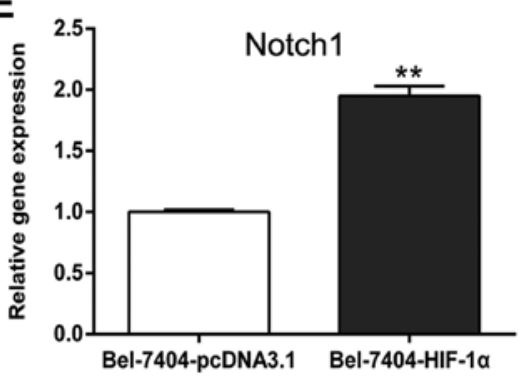

$\mathbf{F}$
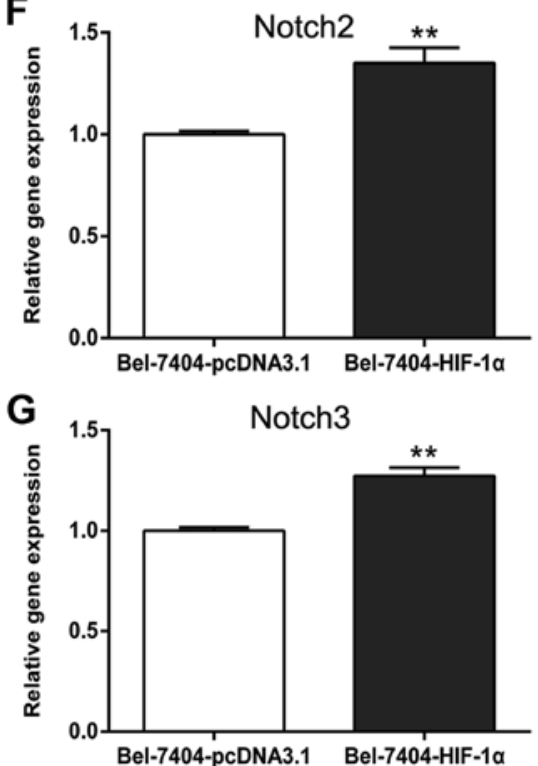

$\mathbf{H}$

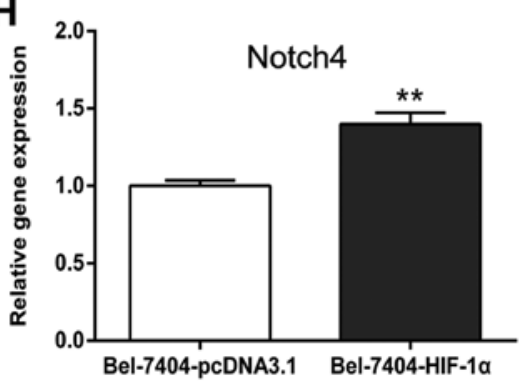

Figure 4. HIF-1 $\alpha$ increases the mRNA expression of Notch1, 2, 3 and 4 in the HCC cell lines. qPCR results show that the mRNA expression of Notch1, 2, 3 and 4 was increased in the (A-D) HepG2 and (E-H) Bel-7404 cells transfected with HIF-1 $\alpha$ expression plasmids compared with their corresponding control cells transfected with an empty vector (pcDNA3.1). Results are presented as the mean $\pm \mathrm{SE}\left({ }^{* *} \mathrm{P}<0.01\right)$.

Notch4. Our results indicated that overexpression of HIF-1 $\alpha$ significantly increased the expression of Notch1, Notch2, Notch 3 and Notch 4 at both the mRNA and protein levels in the HepG2 and Bel-7404 cells (Figs. 4 and 5). Using the TRANSFAC software, multiple potential HIF-1 $\alpha$ binding sites were identified in the promoter regions of Noth1-4 (Fig. 6). HIF-1 $\alpha$ may enhance the expression of Notch1-4 through binding with the hypoxia response elements (CGTG) in their promoter regions. Therefore, HIF-1 $\alpha$ may be another factor that leads to the increased expression of Notch1, Notch3 and Notch4 in HCC tissues.

\section{Discussion}

Notch receptors are large single-pass type I transmembrane proteins, which are composed of the Notch extracellular domain (NECD), the transmembrane domain and the Notch intracellular domain (NICD) (19). The NECD contains 29-36 tandem epidermal growth factor-like (EGF-like) repeats. Human Notch1 and Notch2 contain 36 EGF-like repeats each, and Notch3 and Notch4 contain 34 and 29 EGF-like repeats, respectively (19). Moreover, NICDs of different Notch receptors have different structures, which may lead to variations in their intracellular functions (19).

Different Notch receptors vary in their expression levels and their relationships with tumors. Zhou et al used the Kaplan-Meier plotter (KM plotter) database to analyze the relationship between the mRNA expression of Notch1-4 and the prognosis in 1,306 patients with ovarian cancer. However they failed to find any significant correlations between the Notch1 and Notch4 mRNA expression and the prognosis of patients with ovarian cancer. However, they found that high Notch2 
A

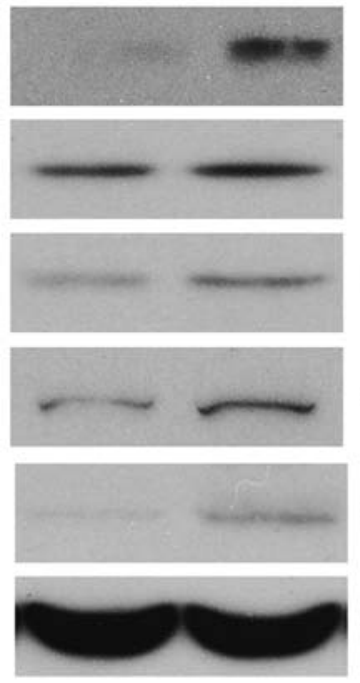

HepG2-pcDNA3.1 HepG2-HIF-1 $\alpha$
B

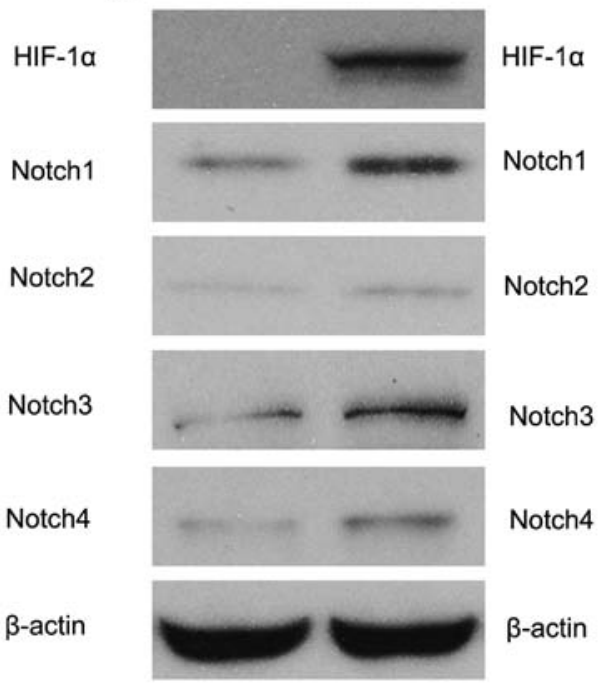

Bel-7404-pcDNA3.1 Bel-7404-HIF-1a

Figure 5. HIF-1 $\alpha$ increases the protein expression of Notch 1-4 in HCC cell lines. Western blotting was used to detect the elevated protein expression of Notch 1-4 in the (A) HepG2 and (B) Bel-7404 cells transiently transfected with HIF-1 $\alpha$ expression plasmids compared to their corresponding control cells transfected with an empty vector (pcDNA3.1). $\beta$-actin was used as a loading control.

A

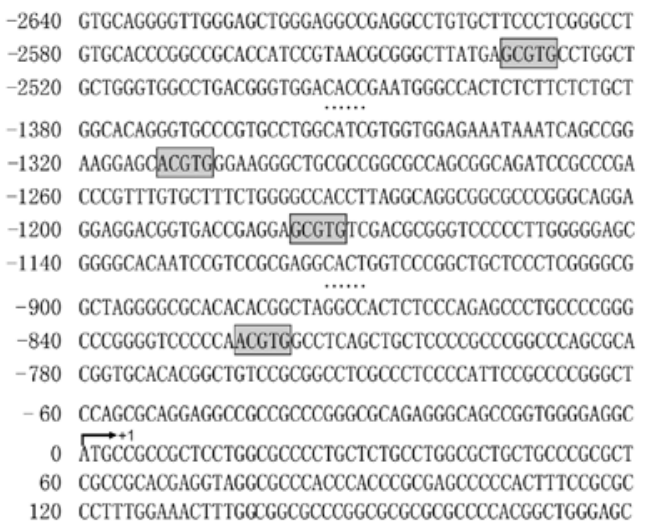
C -1980 GAGGGAGCTGCAGGAAGCCTGTGCAGCATGACTGATTTGGGAAAAGGCAA -1920 TTGATCAAGGCTGGGCGTGGTGGCTCACGCCTGTAATCCCAGCACTTTGG -1860 GAGGCTGAGGCGGGCGGATCACTTGAAGTCAGGAGTTCGAGACCAGCCTG -1020 AgTGGGTTGCTTTCTGGGAGGGCTGAGAGCAAGAAGAGTTTGTGTGTGCA -960 TGTGTGAACACACACACATGCATGTGACTCCTAGTACGTGTCTGAGGTCT -900 GAGGCTGCAAATGTAGTCCTAGGCTCCTGGCCTGGCTGAGTGAGTTTTAG -720 TGGGTTGAAGCGATTTTCCTGCTTCAGCCTCTCGAATAGCTGGGACTACA -660 GGCGTCGCCACCACGCTCAGCTAATTTTTGTATTTTCAGTAGAGTCOGG -600 GTTTCACCATGTTGGCCAGGCTGGTCTCCAACTCTTGACCTCAAGTGATC -420 AAGCTTGGTGCATGGGCTTGCATTTGTGCGTCCGTGGCTGTGGGTCCATG -360 AGCCTCTCAGGACGTCACTGGCCTCAGTTTCCAGAGTTTCTGGGAGGCTG -300 TGTTTTTTGTCCCGGCTCCAGAGGTGTCCGGCTCTGGGTGTGTACTGGGG -240 GATGGGGATGGGGT GCGTGGGCGTTCACGAGGTTGGGTGTGCCCGCCACT -180 CCGGGTTCTGCCCGCGTCTCACTGCATGCTCGGCCTGGGTTTCCGAGGGT $0 \overrightarrow{\text { TTCGAGGGCTGGGGGCGGGGCGGCCOGGCCGCCTCACTTCGGCGAAGTTG }}$ 60 GCGGCGCGGAGGCTGGCCCGGGACGCGOCCGGAGCCCAGGGAMGGAGGGA
B

-4140 GTTTTTGGAAGGTCTAAATCACTGCGCAACTGCAAGGCATTATTAAAAGA -4080 A MTCTTCCTTTTAATTCCTGCTTCCACAAACATCTCATCACATATCCTTG -4020 GGTTCCTGGGTTACATGGAGAGATTCGTGGTGTAGCTCAATCTATGTGTC -3960 ATAGCTATCCCACAATCTCTTCTTCAACTTCTCCAACACACCAAGACATT -3900 CTTCCAGTCTTGCATTGTTCCCCAGTTTATATAGATATATATGTTCCTCA -600 TATCATCCCACTGCCAAGCACACAGCTACGCAAAGTAAGGCACTTAAGAA -540 ATATTCGCTACACGGATGAACAAATGAATGAATGAATGGGGCAGCACCAC -480 TOCGTGATGGCTCTATTCCTTTTTAATGGACTTTGGCGCCTTGGCCCACT -420 TTCCTTCTTTGACAGCACTGGTCCTACTCCAGAGCATTCCTCTAGCTAGC -360 AAAGCAGTGTGTGGATGTGTGGGTGTAGGTGGCGATACAGACCTCACTGG -60 CCTGGGTGCCCAAGGCGGCGGGGCCGAGGCCGCGGCGACAGTGGGGCGGG

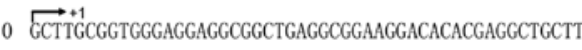
60 CGTTGCACACCCGAGAAAGTTTCAGCCAAACTTCGGGCGGCGGCTGAGGC 120 GGCGGCCGAGGAGCGGCGGACTCGGGGCGCGGGGAGTCGAGGCATTTGCG 180 CCTGGGCTTCGGAGCGTAGCGCCAGGGCCTGAGCCTTTGAAGCAGGAGGA

D

\section{Notch4}

-3180 aCAaGCCCTTCAGGTGAATCTGATCCAGACTCGTTTGaGaAaACCACTGC -3120 TAGGCCGGGCGTGGTGGCTCACGCCTGTAATCCCAGCACTTTGGGAGGCC -3060 AAGGCGGGTGGATCACAAGGTCAGGAGATCGAGACCATCCTGGCTAACAC -3000 AGTGAAACCCCGTCTCTACTAAAAATACAAAAAATTAGCCGGGCGTGGTG -1800 GGACCACACOCTTGATGCTGGCTGCCAGGCTGGCGGTGGAAGACCTGGTT -1740 GAAGAACTGATTGCAGCCCAAGCAGACGTGGGGGCAGAGATAAATGGGG - 1680 TATGTAGAGGAaGGGGTGATGTATGCTATAGAGAaGTTGAGCAGATGGGG -1620 TGGGAGATAGCGTGCAAAATATAGGTGCAGCAGAGGGGCATTCCCTCTCA -900 GGAAAGTGCGGTAACCCGCAAAGCCTGA... -840 GCAAGTGAAGGCAGAAAGGCCCAGTCCTGTGGGCGTGGCCTTCCCTGATA - 780 TCGGCCCTGGCTCTTCTGTACAGGAGCAGACGCCGCTATTCCTGGCGGCG - 540 GAGGCOCGTCACAAAGCCACGCOGGGCCGCGAGGCTGGGCCCTTCCCGCG -480 CGCACGGACGGTGTCAGTAAGCGTG CCCCCGCATGGGGGGGGGCTCTGC 420 CGCGCTGCCGGACGCTGTCAGCCGGAGCAGGCCCTCGTGGGGGCGGAGCT

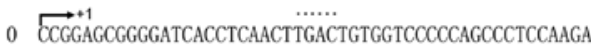

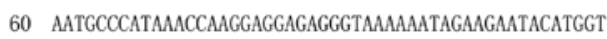

Figure 6. Putative HIF-1 $\alpha$ binding sites in the promoters of human (A) Notch1, (B) Notch2, (C) Notch3 and (D) Notch4. The number indicates the location of the nucleotide relative to the start site of the first exon, which is marked with an arrow. The shaded nucleotides represent the putative hypoxia response elements (CGTG), which are HIF-1 $\alpha$ binding sites that were identified by the TRANSFAC software (http://www.biobase.international.com).

mRNA expression predicted a poor prognosis, while high Notch3 mRNA expression predicted a good prognosis (16).
Xu et al analyzed the correlation between the mRNA expressions of Notch1-4 and the prognosis in 3,554 patients with 
breast cancer using the KM-plotter database. Their results showed that a high expression of Notch1 messenger RNA (mRNA) was correlated with a worse overall survival (OS) in progesterone receptor-negative breast cancer patients. High expression of Notch2, Notch3 and Notch4 mRNA was found to be correlated with a better OS for all breast cancer patients (17).

The Notch1 signaling pathway is one of the most studied pathways involved in the genesis of these tumors, but there is no consensus if it acts as a tumor suppressor or oncogene $(4,5,20,21)$. Various studies confirmed that the Notch1 receptor has a tumor-suppressor function in hepatocellular carcinoma (HCC). Qi et al showed that the Notch1 receptor mediates growth inhibition and apoptosis of HCC-derived cell lines. Overexpression of the Notch1 receptor in these cells induced apoptosis by affecting the balance between p53 and B-cell lymphoma-2 (Bcl-2) (22). The Notch1 receptor also inhibits the proliferation of HCC cells by modifying the expression of the cell cycle regulator, which suggests that the Notch1 receptor takes the role of a tumor suppressor (22). In contrast, other reports found indicators for an oncogenic role of the Notch1 receptor in HCC. This was supported by a recent study, which showed that the inhibition of the Notch1 receptor reduced the HCC rate (20). Furthermore, Wang et al found that a high Notch1 expression in metastatic HCC was significantly correlated with lymph node metastasis (21). Notch1 knockout resulted in an obvious reduction of the metastatic activity of tumor cells in mice, indicating the oncogenicity of Notch1 in HCC (21). The present study revealed that Notch1 was upregulated in liver cancer with respect to precancerous lesions and normal liver tissues. However, no explicit connections were identified between Notch1 and clinical parameters of liver cancer.

The role of Notch2 in HCC has been rarely studied. Gao et al showed that Notch2 was downregulated in HCC tissue compared with the expression level in adjacent nontumor liver tissue (14). Hayashi et al found that Notch2 was significantly associated with an advanced clinical staging of primary HCCs (23). In addition, an in vitro cytological study found that Notch 2 promoted the invasiveness and metastasis of HCC (23), confirming the important role of Notch2 in the occurrence and progression of HCC. Our results did not show any significant differences in Notch2 expression in liver cancer, precancerous cirrhotic liver, and normal liver tissues. Furthermore, Notch2 was not particularly correlated with vascular involvement.

Numerous studies are concerned with the role of Notch3 in HCC. Gao et al did not find any differences in the Notch3 expression between $\mathrm{HCC}$ and adjacent non-tumor liver tissue (14). In contrast, Gramantieri et al found that Notch3 was significantly upregulated in $\mathrm{HCC}$ tissue with respect to precancerous lesions (24). Similar to Gramantieri et al, we found that Notch3 was upregulated in HCC (24). In addition, Notch3 was closely associated with vascular involvement. High expression of Notch3 usually indicates a higher risk of vascular involvement in $\mathrm{HCC}$, which is consistent with the report of $\mathrm{Hu}$ et al (25).

The relationship between Notch 4 and HCC is comparatively less frequently studied. Gramantieri et al compared the expression levels of Notch4 in HCC tissue and precancerous lesions (24). The Notch4 receptor was upregulated in the HCC tissues, whereas the HCC mRNA expression was not significantly different between HCC tissues and precancerous lesions. Ahn et al found that a high Notch4 expression predicted a poor prognosis (26). We also found that Notch4 protein and mRNA were upregulated in $\mathrm{HCC}$ tissues with respect to precancerous lesions, but no connections were revealed between Notch4 and clinical parameters of HCC.

Our results basically agree with previous findings on Notch1-4 expression in liver cancer, albeit with some variations. HCC from different etiologies vary in terms of gene expression (27), which will not be reflected by our results, as we exclusively studied HBV-related liver cancers. This may explain the slight differences to other studies.

The hepatitis B virus is a common pathogen of HCC. HBx, the encoded protein of hepatitis $\mathrm{B}$, is closely associated with the development of HCC (27). HBx can act as a growth factor, promote cell growth, induce cell cycle arrest, interfere with DNA repair, regulate cell proliferation and apoptosis, and cause drug resistance (28). Gao et al reported that HBx upregulated the expression of Notch1 and Notch4 (7), whereas Xu et al showed that HBx inhibited the Notch1 signaling pathway. Confirming the conclusions of Gao et al, we found that HBx upregulated Notch1, Notch3 and Notch4 expression (7), but had no impact on the expression of Notch2. However, the reason for this is unknown.

HIF is a transcriptional factor that is closely related to the occurrence and progression of liver cancer (29). Hypoxia induces the accumulation and entry of HIF- $1 \alpha$ into the nuclei where it binds to HIF-1 $\beta$, forming the active transcription factor. HIF-1 $\alpha$ can bind to core DNA sequence 5'-CGTG-3' within the hypoxia response element (HRE) of target gene promoters, thus promoting gene transcription (29). It is reported that HIF-1 $\alpha$ can upregulate the expression of Notch1 (10-12). We found that in addition to Notch1, HIF-1 $\alpha$ also upregulated Notch2, Notch3, and Notch4. Moreover, several potential HIF-1 $\alpha$ binding sites were found in the promoter regions of Notch1-4 genes. Thus, Notch1-4 may all act as downstream targets of HIF-1 $\alpha$.

By studying the effects of HBx and HIF-1 $\alpha$ on the expression of Notch receptors, we attempted to explain the upregulation of Notch1, Notch3 and Notch4 in liver cancer tissues with respect to precancerous lesions. We detected the expression of Notch1-4 in liver cancer tissues and analyzed their correlations with liver cancer. We believe that Notch3 is more closely related to invasiveness and metastasis of liver cancer. However, the relationship between Notch1-4 and prognosis should be further studied by patient follow-up. Since the findings on the relationship of Notch1-4 and HCC vary from one study to another, more large-sample-size multicenter clinical trials are needed before focusing on Notch1-4 as therapeutic targets.

\section{Acknowledgements}

This study was supported by the Fundamental Research Funds for the Central Universities: the Independent Innovation Fund of Huazhong University of Science and Technology (HUST: 2016YXMS241) and the 2016-2017 Special Fund for the Medical Colleges of Health and Family Planning of Hubei Province (WJ2016-YZ-10). 


\section{References}

1. Chiba T, Iwama A and Yokosuka O: Cancer stem cells in hepatocellular carcinoma: Therapeutic implications based on stem cell biology. Hepatol Res 46: 50-57, 2016.

2. Borggrefe T, Lauth M, Zwijsen A, Huylebroeck D, Oswald F and Giaimo BD: The Notch intracellular domain integrates signals from Wnt, Hedgehog, TGF $\beta / B M P$ and hypoxia pathways. Biochim Biophys Acta 1863: 303-313, 2016.

3. Takebe N, Miele L, Harris PJ, Jeong W, Bando H, Kahn M, Yang SX and Ivy SP: Targeting Notch, Hedgehog, and Wnt pathways in cancer stem cells: Clinical update. Nat Rev Clin Oncol 12: 445-464, 2015.

4. Yap LF, Lee D, Khairuddin A, Pairan MF, Puspita B, Siar CH and Paterson IC: The opposing roles of NOTCH signalling in head and neck cancer: A mini review. Oral Dis 21: 850-857, 2015

5. Gil-García B and Baladrón V: The complex role of NOTCH receptors and their ligands in the development of hepatoblastoma, cholangiocarcinoma and hepatocellular carcinoma. Biol Cell 108: 29-40, 2016.

6. Kongkavitoon P, Tangkijvanich $\mathrm{P}$, Hirankarn $\mathrm{N}$ and Palaga T: Hepatitis $B$ virus $H B x$ activates Notch signaling via deltalike 4/Notch1 in hepatocellular carcinoma. PLoS One 11: e0146696, 2016.

7. Gao J, Xiong Y, Wang Y, Wang Y, Zheng G and Xu H: Hepatitis B virus $X$ protein activates Notch signaling by its effects on Notch1 and Notch4 in human hepatocellular carcinoma. Int J Oncol 48: 329-337, 2016.

8. Sun Q, Wang R, Luo J, Wang P, Xiong S, Liu M and Cheng B: Notch1 promotes hepatitis $\mathrm{B}$ virus $\mathrm{X}$ protein-induced hepatocarcinogenesis via Wnt/ $\beta$-catenin pathway. Int J Oncol 45 : $1638-1648,2014$

9. Xu J, Yun X, Jiang J, Wei Y, Wu Y, Zhang W, Liu Y, Wang W, Wen $\mathrm{Y}$ and $\mathrm{Gu} \mathrm{J}$ : Hepatitis B virus $\mathrm{X}$ protein blunts senescence-like growth arrest of human hepatocellular carcinoma by reducing Notch1 cleavage. Hepatology 52: 142-154, 2010.

10. Tian Q, Xue Y, Zheng W, Sun R, Ji W, Wang X and An R: Overexpression of hypoxia-inducible factor $1 \alpha$ induces migration and invasion through Notch signaling. Int J Oncol 47: 728-738, 2015.

11. Pistollato F, Rampazzo E, Persano L, Abbadi S, Frasson C, Denaro L, D'Avella D, Panchision DM, Della Puppa A, Scienza R, et al: Interaction of hypoxia-inducible factor- $1 \alpha$ and Notch signaling regulates medulloblastoma precursor proliferation and fate. Stem Cells 28: 1918-1929, 2010.

12. Moriyama $H$, Moriyama M, Isshi $H$, Ishihara $S$, Okura $H$, Ichinose A, Ozawa T, Matsuyama A and Hayakawa T: Role of notch signaling in the maintenance of human mesenchymal stem cells under hypoxic conditions. Stem Cells Dev 23: 2211-2224, 2014.

13. Hu JL, Liu LP, Yang SL, Fang X, Wen L, Ren QG and Yu C: Hepatitis $B$ virus induces hypoxia-inducible factor- $2 \alpha$ expression through hepatitis B virus X protein. Oncol Rep 35: 1443-1448, 2016.

14. Gao J, Song Z, Chen Y, Xia L, Wang J, Fan R, Du R, Zhang F, Hong L, Song J, et al: Deregulated expression of Notch receptors in human hepatocellular carcinoma. Dig Liver Dis 40: 114-121, 2008 .
15. Yang SL, Yu C, Jiang JX, Liu LP, Fang X and Wu C: Hepatitis B virus $X$ protein disrupts the balance of the expression of circadian rhythm genes in hepatocellular carcinoma. Oncol Lett 8: 2715-2720, 2014

16. Zhou X, Teng L and Wang M: Distinct prognostic values of four-Notch-receptor mRNA expression in ovarian cancer. Tumour Biol 37: 6979-6985, 2016.

17. Xu J, Song F, Jin T, Qin J, Wu J, Wang M, Wang Y and Liu J: Prognostic values of Notch receptors in breast cancer. Tumour Biol 37: 1871-1877, 2016.

18. Liu Q, Chen J, Liu L, Zhang J, Wang D, Ma L, He Y, Liu Y, Liu Z and $\mathrm{Wu} \mathrm{J}$ : The $\mathrm{X}$ protein of hepatitis $\mathrm{B}$ virus inhibits apoptosis in hepatoma cells through enhancing the methionine adenosyltransferase 2A gene expression and reducing S-adenosylmethionine production. J Biol Chem 286: 17168-17180, 2011.

19. Previs RA, Coleman RL, Harris AL and Sood AK: Molecular pathways: Translational and therapeutic implications of the Notch signaling pathway in cancer. Clin Cancer Res 21: 955-961, 2015.

20. Villanueva A, Alsinet C, Yanger K, Hoshida Y,Zong Y, Toffanin S, Rodriguez-Carunchio L, Solé M, Thung S, Stanger BZ, et al: Notch signaling is activated in human hepatocellular carcinoma and induces tumor formation in mice. Gastroenterology 143: 1660-1669.e7, 2012.

21. Wang XQ, Zhang W, Lui EL, Zhu Y, Lu P, Yu X, Sun J, Yang S, Poon RT and Fan ST: Notch1-Snail1-E-cadherin pathway in metastatic hepatocellular carcinoma. Int J Cancer 131: E163-E172, 2012.

22. Qi R, An H, Yu Y, Zhang M, Liu S, Xu H, Guo Z, Cheng T and Cao X: Notch1 signaling inhibits growth of human hepatocellular carcinoma through induction of cell cycle arrest and apoptosis. Cancer Res 63: 8323-8329, 2003.

23. Hayashi Y, Osanai M and Lee GH: NOTCH2 signaling confers immature morphology and aggressiveness in human hepatocellular carcinoma cells. Oncol Rep 34: 1650-1658, 2015.

24. Gramantieri L, Giovannini C, Lanzi A, Chieco P, Ravaioli M, Venturi A, Grazi GL and Bolondi L: Aberrant Notch3 and Notch4 expression in human hepatocellular carcinoma. Liver Int 27: 997-1007, 2007.

25. Hu L, Xue F, Shao M, Deng A and Wei G: Aberrant expression of Notch3 predicts poor survival for hepatocellular carcinomas. Biosci Trends 7: 152-156, 2013.

26. Ahn S, Hyeon J and Park CK: Notch1 and Notch4 are markers for poor prognosis of hepatocellular carcinoma. Hepatobiliary Pancreat Dis Int 12: 286-294, 2013.

27. Yang SL, Liu LP, Sun YF, Yang XR, Fan J, Ren JW, Chen GG and Lai PB: Distinguished prognosis after hepatectomy of HBV-related hepatocellular carcinoma with or without cirrhosis: A long-term follow-up analysis. J Gastroenterol 51: 722-732, 2016.

28. Geng M, Xin X, Bi LQ, Zhou LT and Liu XH: Molecular mechanism of hepatitis $B$ virus $X$ protein function in hepatocarcinogenesis. World J Gastroenterol 21: 10732-10738, 2015.

29. Lin D and $\mathrm{Wu} \mathrm{J}$ : Hypoxia inducible factor in hepatocellular carcinoma: A therapeutic target. World J Gastroenterol 21: 12171-12178, 2015. 